\title{
Efficacy of preoperative brachytherapy for controlling vaginal bleeding in early-stage cervical cancer: a retrospective study
}

\author{
Quanhong Ping ${ }^{1 \#}$, Jing Zeng ${ }^{1 \#}$, Peisong Sun ${ }^{1}$, Pengpeng $\mathbf{Q u}^{1}$, Shan Jiang ${ }^{2}$, Yuanjing $\mathrm{Hu}^{1}$ \\ ${ }^{1}$ Department of Gynecologic Oncology, Tianjin Central Hospital of Gynecology and Obstetrics, Affiliated Hospital of Nankai University, Tianjin, \\ China; ${ }^{2}$ School of Mechanical Engineering, Tianjin University, Tianjin, China \\ Contributions: (I) Conception and design: Q Ping, J Zeng; (II) Administrative support: Y Hu; (III) Provision of study materials or patients: P Sun; \\ (IV) Collection and assembly of data: P Qu; (V) Data analysis and interpretation: S Jiang; (VI) Manuscript writing: All authors; (VII) Final approval \\ of manuscript: All authors. \\ "These authors contributed equally to this work. \\ Correspondence to: Yuanjing Hu. Department of Gynecologic Oncology, Tianjin Central Hospital of Gynecology and Obstetrics, Affiliated Hospital of \\ Nankai University, Nankaisanmalu Road No. 156, Tianjin 300100, China. Email: huyuanjingzxfck@163.com.
}

Background: In our clinical study, $11 \%$ of the patients with early-stage cervical cancers had different degrees of vaginal bleeding, which required a preoperative intervention. We set to assess the efficacy and safety of preoperative high-dose rate vaginal ovoid brachytherapy (HDR-VOBT) for the treatment of vaginal bleeding in women with early cervical cancer.

Methods: We retrospectively identified and reviewed patients with vaginal bleeding and early-stage cervical carcinoma, treated between January 2011 and December 2014 (median follow-up of 69 months). Of the 116 patients, 59 received preoperative HDR-VOBT (a dose of 8 Gy at $0.5 \mathrm{~cm}$ from the tumor surface), and 57 received traditional vaginal packing with gauze alone, followed by radical hysterectomy and lymphadenectomy. Analysis of the clinical parameters was performed using the chi-square test. The outcome measures were the 3 - and 5 -year survival and the complication rate.

Results: From the 116 patients, 25 had stage IB1, 49 had stage IB2, and 42 had stage IIA1 cervical cancer. No differences were observed regarding the 5-year overall survival (OS) (91.5\% vs. 91.2\%) and diseasefree survival (DFS) (76.3\% vs. 86\%) between the preoperative HDR-VOBT group and the vaginal packing with gauze group. The mean volume of blood loss due to vaginal bleeding after treatment was 83.4 (range: 30-150) mL: 56.8 (range: 30-80) $\mathrm{mL}$ in the preoperative HDR-VOBT group and 111.1 (range: 80-150) $\mathrm{mL}$ in the gauze packing group $(\mathrm{P}<0.001)$. The mean time to achieve hemostasis was $3.5 \mathrm{~h}$ in the preoperative HDR-VOBT group and $8.1 \mathrm{~h}$ in the vaginal packing with gauze group $(\mathrm{P}<0.001)$. There was no significant difference in postoperative risk factors, complications, and survival between the two groups.

Conclusions: Preoperative HDR-VOBT with a dose of $8 \mathrm{~Gy}$ at $0.5 \mathrm{~cm}$ from the surface of the tumor has a better hemostatic effect than vaginal packing with gauze, with no additional complications and no need for adjuvant treatment after the operation.

Keywords: Brachytherapy; cervical cancer; radiotherapy

Submitted Mar 16, 2021. Accepted for publication Jun 16, 2021.

doi: $10.21037 /$ tcr-21-467

View this article at: https://dx.doi.org/10.21037/tcr-21-467

\section{Introduction}

Cervical cancer is the 4th most common type of cancer affecting women worldwide $(1,2)$. More than 14,480 new cases and 4,290 deaths occurred in America in 2021 (3).
Vaginal bleeding is the immediate cause of death in $6 \%$ of women with advanced cervical cancer (4). Due to the availability of effective prevention and screening methods, the incidence and mortality rates associated with cervical 
cancer have declined over time (5). Furthermore, an increased number of patients undergo screening for the early detection of cervical cancer, leading to an increasing proportion of early-stage cervical cancer diagnoses (6). The current National Comprehensive Cancer Network guideline recommends two initial treatment options for early-stage cervical cancers with preoperative clinical International Federation of Gynecology and Obstetrics (FIGO) stages IB1, IB2, and IIA1: radical hysterectomy and lymphadenectomy. Definitive radiation is used with brachytherapy and usually with concurrent chemotherapy (7). In our clinical study, 11\% of the patients with early-stage cervical cancers had different degrees of vaginal bleeding, which required a preoperative intervention. Various studies using high-dose rate vaginal ovoid brachytherapy (HDR-VOBT) reported that this treatment protocol has a strong hemostatic effect, has mild side effects, and can be conveniently used to control bleeding in women with advanced cervical cancer (8-10). However, whether this approach can be employed to control vaginal bleeding in women with early-stage cervical cancer remains unknown. There is a lack of relevant studies investigating the incidence of bleeding in patients with early-stage cervical cancer, the effect of bleeding on treatment results, and the risk of anemia. The present study aimed to investigate the efficacy and safety of preoperative HDR-VOBT for the treatment of vaginal bleeding in women with early-stage cervical cancer.

We present the following article in accordance with the STROBE reporting checklist (available at https://dx.doi. org/10.21037/tcr-21-467).

\section{Methods}

Between January 2011 and December 2014, 1,022 patients with FIGO 2009 stage IB-IIA cervical cancer underwent radical hysterectomy and lymphadenectomy at our Institute. Of them, 121 required a preoperative intervention for various degrees of vaginal bleeding. We explained the pros and cons of preoperative HDR-VOBT and traditional vaginal packing with gauze alone. Treatments were selected based on patients' preference. We reviewed all available medical data. Patients aged 18-70 years, who underwent preoperative cervical biopsy to diagnose squamous carcinoma or adenocarcinoma, underwent radical hysterectomy and lymphadenectomy (by laparotomy or laparoscopy) with preoperative HDR-VOBT or traditional vaginal packing with gauze, and experienced bleeding before treatment (total blood loss: $\leq 200 \mathrm{~mL}$ ) were included in the study. Those who underwent primary cone biopsy, who required a crossover treatment, and whose interval between the completion of treatment and the beginning of the study was less than 6 months were excluded. The study was conducted in accordance with the Declaration of Helsinki (as revised in 2013). The study was approved by the Ethics Review Board of Tianjin Central Hospital of Gynecology and Obstetrics (No. 2020KY098), and informed consent was obtained from all patients.

Five patients who required crossover treatment including emergency interventions (such as surgery or arterial embolization), preoperative HDR-VOBT, and traditional vaginal packing with gauze were excluded from this study. By contrast, 116 patients who underwent preoperative HDR-VOBT or traditional vaginal packing with gauze alone, followed by radical hysterectomy and lymphadenectomy, at our institute were included in the study. For this study, all patients were staged according to the FIGO 2009 staging system (11).

After achieving hemostasis, we inspected the tail of the filling gauze exposed outside the vulva for the presence of bloodstains. If the outer part of the gauze was stained with blood, this indicated that the inner part of the gauze was soaked with blood, and continuous bleeding was expected; hence, the vaginal gauze was replaced, the amount of blood loss was calculated, and the bleeding time was recorded. The volume of blood loss was calculated according to the difference in the mass of the vaginal gauze before and after it was soaked with blood. The volume of blood loss $(\mathrm{mL})$ was equal to the difference between the mass $(\mathrm{g})$ of the gauze before and after use divided by 1.05 . If the tail of the gauze remained unstained after 1 hour, the gauze was removed and the patient was observed for active bleeding. If bleeding was not observed, the time that the patient achieved hemostasis was recorded.

HDR-VOBT was performed using an iridium-192 source with a nominal activity of $10 \mathrm{Ci}$. A vaginal applicator with two ovoids was placed in the vagina against the cervix. During insertion, the gauze was used to temporarily fix the applicator in the vagina. For hemostasis, a dose of $8 \mathrm{~Gy}$ was delivered at $0.5 \mathrm{~cm}$ posterior to the surface of the tumor. After a mean period of 3 days following HDRVOBT, the patients underwent surgery. All patients underwent radical hysterectomy and lymphadenectomy by laparotomy or laparoscopy. Lymphadenectomy included the removal of external iliac lymph nodes, with the intraoperative pathological examination of cryosections. If lymph node metastasis was observed, lymphadenectomy 
would be extended to the common iliac lymph nodes. Paraaortic lymph node dissection was performed in cases with lymphadenopathies noted during clinical examination or on preoperative imaging.

Adjuvant treatment was performed in patients with lymph node metastasis and/or parametrial invasion or in those with intra-tumoral vascular invasion. The treatment included external pelvic radiotherapy with or without concomitant platinum-based chemotherapy. The pelvic dose applied postoperatively did not consider the dose delivered through HDR-VOBT to the parametrium and the external iliac lymph nodes.

Follow-up visits were scheduled at 3 months after the completion of treatment, every 6 months after 5 years, and once a year after the first 5 years. Imaging examinations, including abdominopelvic magnetic resonance imaging (MRI) and computed tomography (CT), were performed at some follow-up visits.

The safety of treatments was assessed based on the prevalence of complications. Early complications, defined as complications occurring within the first 2 months after the surgical procedure, and late complications, occurring after the first 2 months, were evaluated according to the Chassagne glossary (12): grade 1, mild complications that may cause some functional impairment; grade 2, moderate complications resulting in intermittent or persistent interference with normal activity; grade 3, severe complications that require surgery or invasive procedures and affect the performance of the patient; and grade 4, death of a patient due to a complication. Regarding the postoperative radiation therapy to the pelvis, acute and chronic toxicities were assessed according to the Radiation Therapy Oncology Group acute and late toxicity criteria (13).

\section{Statistical analysis}

The overall survival (OS) and disease-free survival (DFS) were calculated using the Kaplan-Meier method. The duration of survival was calculated from the initiation of treatment. The endpoint of OS was death, while that of DFS was local recurrence, metastasis, or death. The clinical parameters were compared using the chi-square test. The medians and life tables were computed using the productlimit estimate with the Kaplan-Meier method, while the log-rank test was used to assess the statistical significance. A $\mathrm{P}$ value below 0.05 was considered significant. All statistical analyses were performed using the SPSS 22.0 software package (IBM, Chicago, IL, USA).

\section{Results}

The median follow-up duration was 69 (range: 1694) months, and the patients' median age was 47 (range: 25-66) years. Approximately $4.3 \%$ of the patients smoked more than 10 cigarettes per day. The median clinical tumor size was 3.1 (range: $1.2-4$ ) cm. Of the 116 patients, $25(21.6 \%)$ were diagnosed with stage IB1 cervical cancer, $49(42.2 \%)$ with stage IB2 cervical cancer, and $42(36.2 \%)$ with stage IIA1 cervical cancer. Based on the results of the preoperative cervical biopsy, 100 patients (86.2\%) had squamous carcinoma, while $16(13.8 \%)$ had adenocarcinoma. A total of 27 patients $(23.3 \%)$ had highgrade tumors, while $89(76.7 \%)$ had intermediate-grade and low-grade tumors. Initial assessments including pelvic ultrasonography and MRI were performed in all patients. Imaging examination showed suspicious lymph nodes in two patients $(1.7 \%)$. However, lymph node metastasis was not confirmed postoperatively. The mean volume of blood loss due to vaginal bleeding prior to treatment was 148 (range: $50-200$ ) $\mathrm{mL}$ : a total of 70 patients $(60.3 \%$ ) had a blood loss of less than or equal to $150 \mathrm{~mL}$, while $46(39.7 \%)$ had a blood loss of greater than $150 \mathrm{~mL}$. Moreover, 59 patients $(50.9 \%)$ received preoperative HDR-VOBT, while 57 (49.1\%) underwent vaginal packing with gauze only (Table 1).

The mean volume of blood loss due to vaginal bleeding after treatment was 83.4 (range: $30-150$ ) mL: 56.8 (range: $30-80) \mathrm{mL}$ in the preoperative HDR-VOBT group and 111.1 (range: $80-150) \mathrm{mL}$ in the gauze packing group $(\mathrm{P}<0.001)$. The mean time to achieve hemostasis was $3.5 \mathrm{~h}$ in the preoperative HDR-VOBT group and $8.1 \mathrm{~h}$ in the vaginal packing with gauze group $(\mathrm{P}<0.001)$ (Table 2$)$.

According to the Querleu-Morrow classification of radical hysterectomy (14), 40 patients underwent laparoscopic interventions [5 patients ( $4.5 \%)$, type B2 interventions; 35 patients (30.2\%), type $\mathrm{C} 2$ interventions]. The other 76 patients $(65.5 \%)$ underwent type C2 laparotomy interventions. The median operative blood loss volume was 466 (range: 100-1,100) mL: 269 (range: $100-500) \mathrm{mL}$ in the laparoscopy group and 570 (range: $200-1,100) \mathrm{mL}$ in the laparotomy group. The median total number of removed lymph nodes was 21 (range: 0-53). Of the total study population, 2 patients $(1.7 \%)$ had parametrial invasion, 58 (50\%) had lymphovascular invasion, and 19 (16.4\%) had pelvic lymph node metastasis (Table 2).

Depending on the presence of high-risk factors (parametrial invasion, lymph node invasion, and positive 
Table 1 Distribution of the patient's clinical parameters in the two treatment groups

\begin{tabular}{|c|c|c|c|c|}
\hline Parameters & Total & Preoperative HDR-VOBT & Gauze packing & $P$ value \\
\hline Mean age [range] & $47[25-66]$ & 46.7 [25-63] & $47[26-66]$ & 0.742 \\
\hline Menopause, n (\%) & $40(34.5)$ & $24(20.7)$ & $16(13.8)$ & 0.153 \\
\hline Mean BMI [range] & $24[15.8-34.6]$ & $24.1[18.4-34.6]$ & $24.2[15.8-34.5]$ & 0.939 \\
\hline Intermediate and low-grade, n (\%) & $89(76.7)$ & $45(38.8)$ & $44(37.9)$ & 0.907 \\
\hline \multicolumn{5}{|l|}{ Histology, n (\%) } \\
\hline Squamous & $100(86.2)$ & $53(45.7)$ & $47(40.5)$ & \\
\hline Adenocarcinoma & $16(13.8)$ & $6(5.2)$ & $10(8.6)$ & 0.367 \\
\hline IB2 & $49(42.2)$ & $26(22.4)$ & $23(19.8)$ & 0.685 \\
\hline$\| \mathrm{A} 1$ & $42(36.2)$ & $21(18.1)$ & $21(18.1)$ & 0.889 \\
\hline Mean volume of bleeding in $\mathrm{mL}$ before treatment [range] & $148[50-200]$ & $142.5[70-200]$ & $154.2[50-200]$ & 0.11 \\
\hline
\end{tabular}

HDR-VOBT, high-dose rate vaginal ovoid brachytherapy; BMI, body mass index; FIGO, International Federation of Gynecology and Obstetrics.

Table 2 Distribution of the patients' treatment parameters in the two treatment groups

\begin{tabular}{|c|c|c|c|c|}
\hline Parameters & Total & Preoperative HDR-VOBT & Gauze packing & $P$ value \\
\hline Median time to achieve hemostasis in hours [range] & $5.8[2-20]$ & $3.5[2-6]$ & $8.1[2-20]$ & $<0.001$ \\
\hline \multicolumn{5}{|l|}{ Type of radical surgery, $\mathrm{n}(\%)$} \\
\hline Laparotomy & $76(65.5)$ & $35(30.2)$ & $41(35.3)$ & \\
\hline Median operation time [type C2] [range] & $180.2[140-265]$ & $182.1[140-265]$ & $178.2[140-240]$ & 0.671 \\
\hline Median operative blood loss volume in $\mathrm{mL}$ [range] & $466[100-1,100]$ & $434.8[100-1,000]$ & $498.3[200-1,100]$ & 0.133 \\
\hline \multicolumn{5}{|l|}{ Chassagne classification of complications, n (\%) } \\
\hline Grade 1 & $47(40.5)$ & $22(19.0)$ & $25(21.6)$ & 0.471 \\
\hline Early complications, n (\%) & $21(18.1)$ & $9(7.8)$ & $12(10.3)$ & 0.417 \\
\hline Late complications, n (\%) & $50(43.1)$ & $21(18.1)$ & $29(25.0)$ & 0.097 \\
\hline \multicolumn{5}{|l|}{ Number of excised pelvic lymph nodes } \\
\hline Median [range] & $21[0-53]$ & $20[1-53]$ & $22[0-43]$ & 0.892 \\
\hline Positive pelvic lymph nodes, n (\%) & $19(16.4)$ & $11(9.5)$ & $8(6.9)$ & 0.503 \\
\hline Parametrial invasion, n (\%) & $2(1.7)$ & $1(0.87)$ & $1(0.87)$ & 0.98 \\
\hline Lymphovascular invasion, $\mathrm{n}(\%)$ & $58(50.0)$ & $32(27.6)$ & $26(22.4)$ & 0.353 \\
\hline
\end{tabular}

HDR-VOBT, high-dose rate vaginal ovoid brachytherapy. 
OS

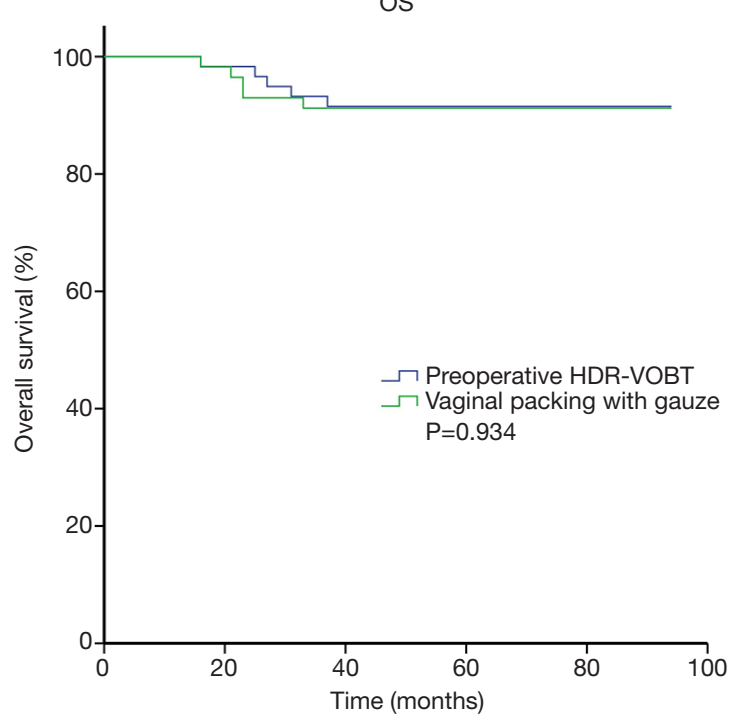

Figure 1 The 5-year OS in the preoperative HDR-VOBT group and the vaginal packing with gauze group. OS, overall survival; HDR-VOBT, high-dose rate vaginal ovoid brachytherapy.

surgical margins), potentially important risk factors (Sedlis criteria), and close surgical margins (below $5 \mathrm{~mm}$ ), 39 patients $(33.6 \%)$ received postoperative adjuvant therapy. Among these patients, 15 (12.9\%) received additional external beam radiation (conformal radiation therapy) without chemotherapy, $4(3.4 \%)$ received postoperative chemotherapy, and $20(17.2 \%)$ received chemoradiotherapy. Three-dimensional conformal radiation therapy was delivered using 6-MV photons with a four-field box technique. Patients underwent CT (16-slice Philips Brilliance Big Bore CT) simulation using oral contrast agents. The clinical target volume (CTV) was contoured on the individual axial CT slice for each patient. The CTV covered the vaginal fragment and regional lymph nodes (the common iliac, internal iliac, external iliac, obturator, presacral, and/or para-aortic lymph nodes). The planning target volume (PTV) was defined as the CTV plus a $7-\mathrm{mm}$ margin. A total dose of 45 Gy (1.8 Gy per fraction) was delivered to the PTV. A docetaxel plus cisplatin regimen was used as treatment for squamous cell carcinoma (135$155 \mathrm{mg} / \mathrm{m}^{2}$, day 1 , and $60 \mathrm{mg} / \mathrm{m}^{2}$, respectively) (area under the concentration-time curve: $5.0-7.5$, day 1 ). The median number of chemotherapy cycles was 4 (range: $1-6)$. In total, 20 patients received four or more cycles of chemotherapy.

The median follow-up duration was 69 (range: 1694) months. None of the patients were lost to follow-up. In

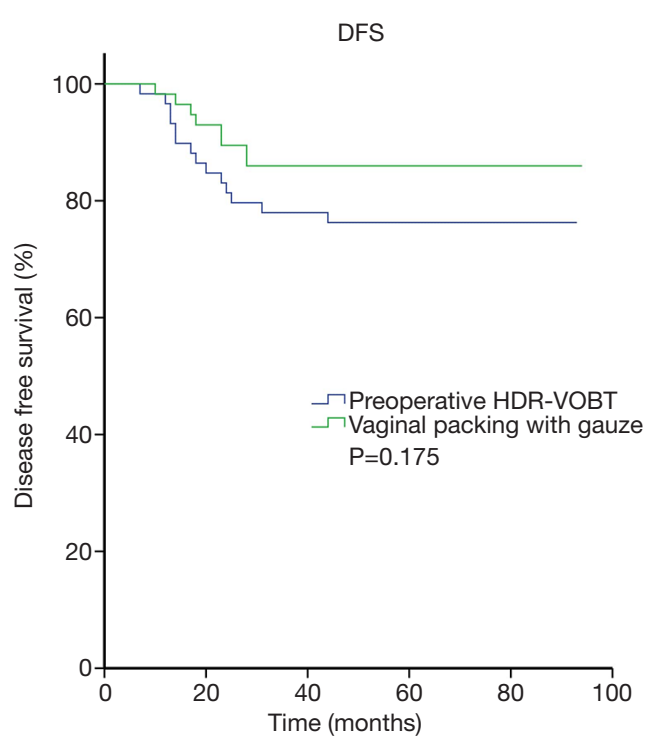

Figure 2 The 5 -year DFS in the preoperative HDR-VOBT group and the vaginal packing with gauze group. DFS, disease-free survival; HDR-VOBT, high-dose rate vaginal ovoid brachytherapy.

total, 8 patients $(5.2 \%)$ who received postoperative radiation therapy with high-risk factors had local recurrence; among them, seven patients experienced parametrial recurrence, while one experienced a bladder recurrence. Ten patients $(9.3 \%)$ experienced metastatic relapse; among them, five patients experienced isolated metastatic relapse, while another five experienced metastatic and local relapse. The 3 -year OS and DFS were $92.2 \%$ and $81.9 \%$, respectively. The 5 -year OS and DFS were $91.4 \%$ and $81 \%$, respectively. As shown in Figures 1,2, no differences were observed in the 5 -year OS (91.5\% vs. $91.2 \%)$ and DFS (76.3\% vs. 86\%) between the preoperative HDR-VOBT group and the vaginal packing with gauze group.

According to the Chassagne classification, $40.5 \%$ of the patients had grade 1 complications, $15.5 \%$ had grade 2 complications, and $2.6 \%$ had grade 3 complications. Table 3 shows the number, type, and grade of the intraoperative, early, and late postoperative complications. In the early postoperative period, one patient underwent surgery to remove the lesions in the ureter and bladder. Early complications (in the first 2 months following surgery) occurred in 21 patients (18.1\%). Among them, 10 patients $(8.6 \%)$ who underwent radical surgery type C2 (2, laparoscopy; 8 , laparotomy) experienced urinary retention (duration of postoperative catheterization: up to 14 days). Five patients (4.3\%) (2, laparoscopy; 3, laparotomy) 
Table 3 Intraoperative, early, and late postoperative complications, according to the Chassagne glossary

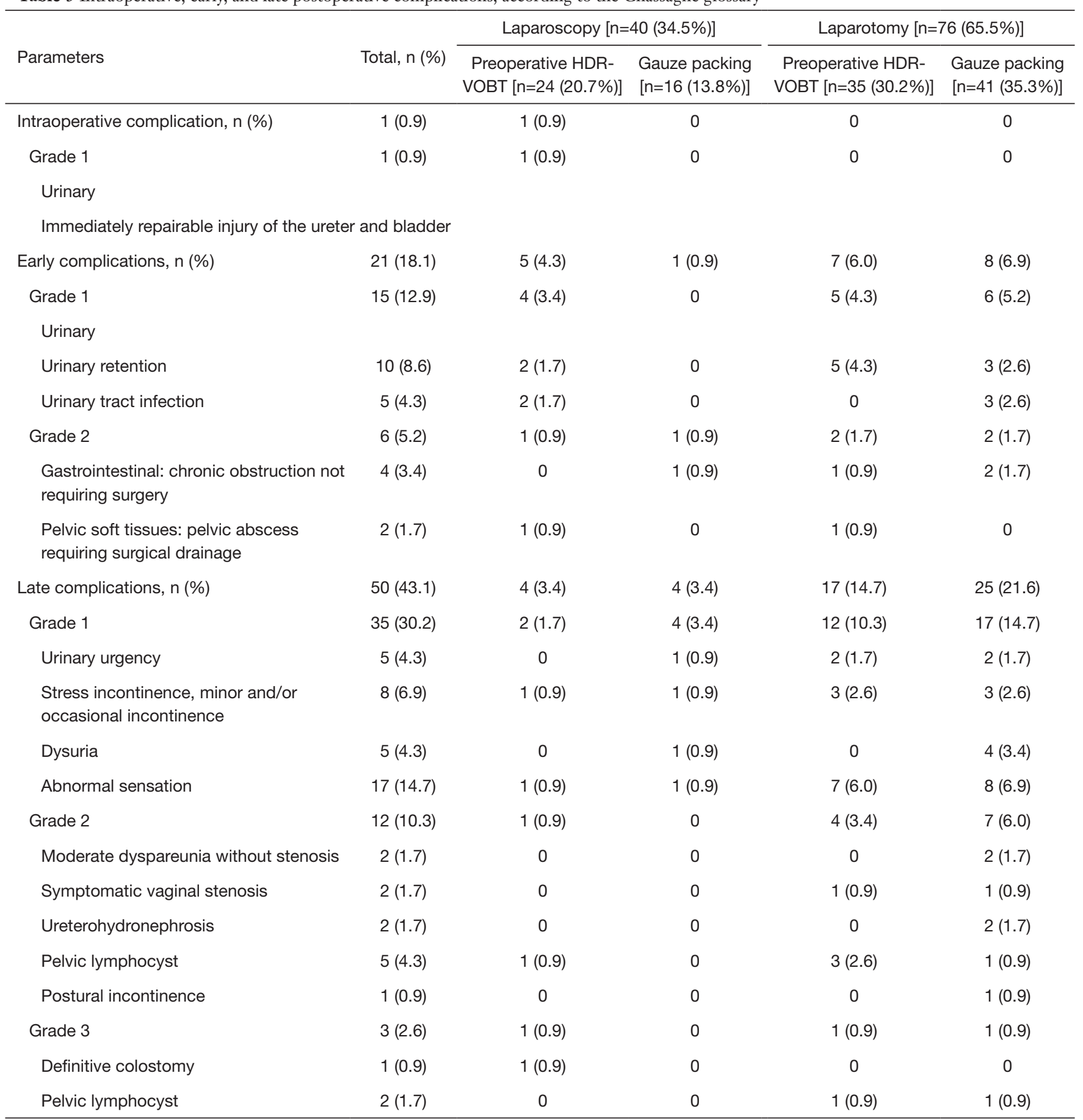

HDR-VOBT, high-dose rate vaginal ovoid brachytherapy.

had urinary tract infections. Four patients underwent gastrointestinal decompression as a management for lower intestinal obstruction and two experienced pelvic seroma requiring transvaginal fine-needle aspiration.
As regards long-term toxicity, 50 patients developed complications: 35 had grade 1 and frequently experienced urinary complications, including urgency, dysuria, and abnormal sensation; 12 had grade 2, including 2 with 
Table 4 Incidence of acute and chronic toxicity associated with postoperative radiation therapy to the pelvis with preoperative HDR-VOBT and gauze packing

\begin{tabular}{|c|c|c|c|c|c|}
\hline Acute/chronic & Parameters & Total $(n=35), n(\%)$ & $\begin{array}{c}\text { Preoperative HDR-VOBT } \\
(\mathrm{n}=19), \mathrm{n}(\%)\end{array}$ & $\begin{array}{c}\text { Gauze packing }(\mathrm{n}=16) \text {, } \\
\mathrm{n}(\%)\end{array}$ & $P$ value \\
\hline \multirow{2}{*}{$\begin{array}{l}\geq \text { Grade } 3 \text { acute } \\
\text { toxicity }\end{array}$} & Hematological toxicity & $11(31.4)$ & $7(36.8)$ & $4(25.0)$ & 0.493 \\
\hline & Urinary toxicity & $4(11.4)$ & $3(15.8)$ & $1(6.3)$ & 0.608 \\
\hline
\end{tabular}

HDR-VOBT, high-dose rate vaginal ovoid brachytherapy.

moderate dyspareunia without stenosis (underwent laparoscopy), 2 with symptomatic vaginal stenosis, 2 with ureterohydronephrosis, 5 with pelvic lymphocysts, and 1 with postural incontinence; and 3 had grade 3, including 1 with definitive colostomy and 2 with pelvic lymphocysts. None of the patients died from post-therapeutic complications (Table 3).

Of 39 patients with high-risk factors, 35 (30.2\%) received postoperative radiation therapy to the pelvis. Among them, 19 patients received preoperative HDR-VOBT, and 16 underwent gauze packing. As shown in Table 4, 11 patients (31.4\%) experienced acute hematological toxicity of grade 3 or higher. The incidence rates were similar between the preoperative HDR-VOBT group (36.8\%) and gauze packing group $(25 \%)$. Seven patients $(20 \%)$ developed acute gastrointestinal toxicity of grade 3 or higher. The incidence rates were similar between the preoperative HDR-VOBT group $(21.1 \%)$ and gauze packing group (18.8\%). Four patients $(11.4 \%)$ experienced acute urinary toxicity of grade 3 or higher. The incidence rates in patients treated with preoperative HDR-VOBT and gauze packing were $15.8 \%$ and $6.3 \%$, respectively $(\mathrm{P}=0.608)$. The incidence rates of chronic gastrointestinal and genitourinary toxicity of grade 3 or higher were $5.7 \%$ and $2.9 \%$, respectively. The chronic gastrointestinal and genitourinary toxicity associated with HDR-VOBT was not significantly higher than that associated with gauze packing. Three patients $(8.6 \%)$ developed grade 3-4 chronic toxicity, including two with proctitis and one with vesicovaginal fistula. The patient with vesicovaginal fistula underwent surgical repair.

\section{Discussion}

Vaginal bleeding is common in women with advanced cervical cancer, with a prevalence of $84.0 \%$ (15). However, persistent vaginal bleeding also occurs in patients with early-stage cervical cancer, which might interfere with the gynecological examination and clinical staging. In our study, $11.8 \%$ of the patients with early-stage cervical cancer presented various degrees of vaginal bleeding. The mean blood loss due to vaginal bleeding before treatment was 148 (range: 50-200) $\mathrm{mL}$. Invasive cervical carcinomas had a mean diameter of $3.1 \mathrm{~cm}$ and were either exophytic (protruding from the surface) or endophytic (with stromal infiltration). Therefore, preoperative measures to control bleeding in women with early-stage cervical cancer are often required. The preoperative treatment should be selected based on the severity of bleeding, the effectiveness of the intervention, and the operative prognosis.

Vaginal packing with gauze rolls is a simple first-aid measure to control bleeding. To perform an effective vaginal packing, it is ideal to expose the upper part of the vagina by using instruments (speculum) with the patient placed in a lithotomy position (with the legs in stirrups). The patients might require sedation or short-term general anesthesia. The vaginal vault is tightly packed in order to maintain the pressure in the vagina. In this situation, catheterization is often required. Owing to the urethral pressure, it is difficult for patients to pass urine. To enhance the effectiveness of the vaginal packing, patients' mobility is often restricted, in the bed with limb elevation (16). Hemostatic agents (with local, per-oral, or parenteral administration) might be helpful for treating persistent vaginal bleeding. During vaginal packing, the underlying infection (often occurring in necrotic tumors) can also cause bleeding.

Radiotherapy is effective for the treatment of vaginal bleeding in patients with cervical cancer. Brachytherapy plays an important role in controlling the bleeding caused by cervical tumors. The North American guidelines recommend two regimens of 5 Gy delivered via a vaginal 
cuff, which have been proven to reduce the need for additional transfusion in $93 \%$ of the patients without acute or long-term grade 3-5 complications (17). The conformal dose distribution can minimize the dose delivered to the rectum and the bladder, without changing the definitive treatment plan. Symptomatic bleeding from a cervical tumor can be reduced by vaginal cuff brachytherapy before the initiation of the definitive treatment (18).

In our center, one regimen of 8 Gy delivered at $0.5 \mathrm{~cm}$ from the surface of the tumor is used to control persistent vaginal bleeding, which has the same biological equivalent dose as that with two regimens of $5 \mathrm{~Gy}$ (19). The volume of blood loss due to vaginal bleeding and the time needed to achieve hemostasis were significantly less in the preoperative HDR-VOBT group than in the vaginal packing with gauze group, indicating that preoperative HDR-VOBT might be more effective in controlling vaginal bleeding than vaginal packing with gauze alone. Preoperative HDR-VOBT could improve the patients' condition, enhance the curative effect, shorten the time of treatment, and reduce the risk of infection.

No significant difference was observed in the intraoperative blood loss and operation time between the two groups. No significant differences were also noted in the incidence of intraoperative, early, and late postoperative complications according to the Chassagne glossary between the two groups. Preoperative HDR-VOBT with a dose of 8 Gy did not increase the incidence of surgical complications. The effect of preoperative brachytherapy on the survival of patients with locally advanced cervical cancer remains controversial, but most studies confirmed that it has no effect on the survival rate of patients with stages IB2 and IIA cervical cancer $(20,21)$. Our study aimed to analyze the efficacy and safety of preoperative HDR-VOBT with a dose of 8 Gy to control vaginal bleeding in patients with early-stage cervical cancer. The dose of radiotherapy used to achieve hemostasis was lower than the dose of radical radiotherapy. No significant difference was observed in the incidence of postoperative complications, incidence of postoperative radiation therapy complications, and OS and DFS between the two groups.

Furthermore, this is a small-sample retrospective study with a certain inherent bias. Brachytherapy patients also underwent brief treatment with vaginal packing before or after radiation, which possibly contributed to the favorable outcomes in this group. The efficacy and safety of preoperative HDR-VOBT with a dose of 8 Gy for controlling vaginal bleeding need to be further evaluated in prospective studies.

\section{Conclusions}

This is the first retrospective study to evaluate the efficacy and safety of HDR-VOBT in controlling vaginal bleeding in patients with invasive early-stage cervical cancer. Our study showed that preoperative HDR-VOBT with a dose of 8 Gy delivered at $0.5 \mathrm{~cm}$ from the surface of the tumor has a better hemostatic effect than traditional vaginal packing with gauze, with no additional complications or requirement for adjuvant treatments.

\section{Acknowledgments}

Funding: This work was supported by the Science and Technology Project of Tianjin (grant No. 18YFZCSY01300) and Health Science and Technology Project of Tianjin (grant No. ZC20111 and KJ20149).

\section{Footnote}

Reporting Checklist: The authors have completed the STROBE reporting checklist. Available at https://dx.doi. org/10.21037/tcr-21-467

Data Sharing Statement: Available at https://dx.doi. org/10.21037/tcr-21-467

Peer Review File: Available at https://dx.doi.org/10.21037/ tcr-21-467

Conflicts of Interest: All authors have completed the ICMJE uniform disclosure form (available at https://dx.doi. org/10.21037/tcr-21-467). The authors have no conflicts of interest to declare.

Ethical Statement: The authors are accountable for all aspects of the work in ensuring that questions related to the accuracy or integrity of any part of the work are appropriately investigated and resolved. The study was conducted in accordance with the Declaration of Helsinki (as revised in 2013). The study was approved by the Institutional Ethics Review Board of Tianjin Central Hospital of Gynecology and Obstetrics (No. 2020KY098), and informed consent was obtained from all patients.

Open Access Statement: This is an Open Access article 
distributed in accordance with the Creative Commons Attribution-NonCommercial-NoDerivs 4.0 International License (CC BY-NC-ND 4.0), which permits the noncommercial replication and distribution of the article with the strict proviso that no changes or edits are made and the original work is properly cited (including links to both the formal publication through the relevant DOI and the license). See: https://creativecommons.org/licenses/by-nc-nd/4.0/.

\section{References}

1. Li Y, Xu C. Human Papillomavirus-Related Cancers. Adv Exp Med Biol 2017;1018:23-34.

2. Cervical cancer. Estimated incidence, mortality and prevalence worldwide in 2018. 2019. Available online: http://globocan.iarc.fr/Pages/fact_sheets_cancer.aspx

3. National Cancer Institute. Surveillance, Epidemiology and End Results Program. Cancer stat facts: cervical cancer. 2021. Available online: https://seer.cancer.gov/statfacts/ html/cervix.html

4. Eleje GU, Eke AC, Igberase GO, et al. Palliative interventions for controlling vaginal bleeding in advanced cervical cancer. Cochrane Database Syst Rev 2019;3:CD011000.

5. Johnson CA, James D, Marzan A, et al. Cervical Cancer: An Overview of Pathophysiology and Management. Semin Oncol Nurs 2019;35:166-74.

6. Marth C, Landoni F, Mahner S, et al. Cervical cancer: ESMO Clinical Practice Guidelines for diagnosis, treatment and follow-up. Ann Oncol 2017;28:iv72-83.

7. National Comprehensive Cancer Network, Clinical Practice Guideline in Oncology. Cervical Cancer. 2018. Available online: https://www.nccn.org/professionals/ physician_gls/pdf/cervical. pdf

8. Grover S, Longo J, Einck J, et al. The unique issues with brachytherapy in low- and middle-income countries. Semin Radiat Oncol 2017;27:136-42.

9. Moelle U, Mathewos A, Aynalem A, et al. Cervical cancer in ethiopia: the effect of adherence to radiotherapy on survival. Oncologist 2018;23:1024-32.

10. Chen CS, Park S, Shin JH, et al. Endovascular treatment for the control of active vaginal bleeding from uterine cervical cancer treated with radiotherapy. Acta Radiol 2018;59:1336-42.

11. Pecorelli S, Zigliani L, Odicino F. Revised FIGO staging for carcinoma of the cervix. Int J Gynaecol Obstet 2009; $105: 107-8$.
12. Chassagne D, Sismondi P, Horiot JC, et al. A glossary for reporting complications of treatment in gynecological cancers. Radiother Oncol 1993;26:195-202.

13. Cox JD, Stetz J, Pajak TF. Toxicity criteria of the Radiation Therapy Oncology Group (RTOG) and the European Organization for Research and Treatment of Cancer (EORTC). Int J Radiat Oncol Biol Phys 1995;31:1341-6.

14. Querleu D, Cibula D, Abu-Rustum NR. 2017 Update on the Querleu-Morrow Classification of Radical Hysterectomy. Ann Surg Oncol 2017;24:3406-12.

15. Tapera O, Dreyer G, Kadzatsa W, et al. Determinants of access and utilization of cervical cancer treatment and palliative care services in Harare, Zimbabwe. BMC Public Health 2019;19:1018.

16. Hay CM, Lefkowits C, Crowley-Matoka M, et al. Gynecologic oncologist views influencing referral to outpatient specialty palliative care. Int J Gynecol Cancer 2017;27:588-96.

17. Suneja G, Brown D, Chang A, et al. American Brachytherapy Society: Brachytherapy treatment recommendations for locally advanced cervix cancer for low-income and middle-income countries. Brachytherapy 2017;16:85-94.

18. Romanyukha A, Carrara M, Mazzeo D, et al. An innovative gynecological HDR brachytherapy applicator system for treatment delivery and real-time verification. Phys Med 2019;59:151-7.

19. Albuquerque K, Hrycushko BA, Harkenrider MM, et al. Compendium of fractionation choices for gynecologic HDR brachytherapy-An American Brachytherapy Society Task Group Report. Brachytherapy 2019;18:429-36.

20. Greggi S, Kenter G, Vergote I, et al. Results from neoadjuvant chemotherapy followed by surgery compared to chemoradiation for Stage IB2-IIB cervical cancer: EORTC55994. International Journal of Gynecologic Cancer 2019;29:A12.

21. Gupta S, Maheshwari A, Parab P, et al. Neoadjuvant chemotherapy followed by radical surgery versus concomitant chemotherapy and radiotherapy in patients with stage IB2, IIA, or IIB squamous cervical cancer: a randomized controlled trial. J Clin Oncol 2018;36:1548-55.

Cite this article as: Ping Q, Zeng J, Sun P, Qu P, Jiang S, Hu Y. Efficacy of preoperative brachytherapy for controlling vaginal bleeding in early-stage cervical cancer: a retrospective study. Transl Cancer Res 2021;10(7):3259-3267. doi: 10.21037/tcr-21-467 